\title{
Diffusion-dominated asymptotics of solution to chemotaxis model
}

\author{
ANDRZEJ RACZYŃSKI
}

Abstract. The paper contains results on the asymptotic behavior, as $t \rightarrow+\infty$, of small solutions to simplified Keller-Segel problem modeling chemotaxis in the whole space $\mathbb{R}^{2}$. We prove that the multiple of the heat kernel is a surprisingly good approximation of solutions.

\section{Introduction}

In this paper, we consider the parabolic-elliptic system in the whole plane $\mathbb{R}^{2}$

$$
\begin{aligned}
u_{t} & =\Delta u-\nabla \cdot(u \nabla v), \\
\Delta v & =v-u, \\
u(x, 0) & =u_{0}(x),
\end{aligned}
$$

which is a simplified version of the parabolic-parabolic system (2.6)-(2.7), called the Keller-Segel model, and describes the movement of amoebae, whose density is denoted by $u(x, t)$, in the presence of a chemoattractant with the concentration given by $v(x, t)$. This simplification was first introduced for bounded domains by $T$. Nagai [24], Jäger and Luckhaus [16] and Wolansky [29] in order to exploit the contrasts between the existence and nonexistence of global in time solutions. The main goal of this paper is to establish the intermediate asymptotics of the solution.

Let us outline the main results concerning the considered model. Since much attention has been paid to the blowup problems for this system, recall that $8 \pi$ is the critical value of initial mass $\left(\int u_{0}(x) d x\right)$ for the existence problem. Namely, below this value, one can construct global solutions to the problem while for mass above $8 \pi$ (with the second momentum $\int|x|^{2} u_{0}(x) d x$ small enough, i.e., smaller than the value of $g(M)$ a monotone increasing function of mass $M$ ), the blowup occurs (see [10, Theorem 1.2], [21, Theorem 2] for the form of $g(M)$ ).

Although the mainstream of research for the whole plane case is devoted to the problem of finite time blowup, the elements of global existence, under smallness assumption on initial mass, can be found in [11,21], and [18]. 
As an application of symmetrization techniques, it was shown in [11, Theorem 3.1] that for nonnegative initial condition $u_{0}(x)$ satisfying $u_{0} \in L^{1}\left(\mathbb{R}^{2}\right) \cap W^{1, p}\left(\mathbb{R}^{2}\right), p>$ 2 , with $L^{1}$ norm bounded by $8 \pi$, there exists a unique nonnegative, global in time solution of (1.1)-(1.3). Additionally, it was proved that for all $p \geq 1$, the $L^{p}$ norms of the solution are bounded by a constant depending on $p$ and $u_{0}$.

Although the paper [21] is mainly devoted to finite time blowup, it was also shown, under smallness assumption on initial mass only, the existence of a mild global in time solution to the problem in a subspace of $L^{1}$. To be precise, not only the $L^{1}$ norm of the solution is bounded, but also the quantity $t^{1-\frac{1}{p}}\|u(t)\|_{p}$ with $p \in\left(\frac{4}{3}, 2\right)$ is controlled for $t \in \mathbb{R}^{+}$.

A related result is obtained in the paper [18], where general type of the aggregation equation, i.e., of the form

$$
u_{t}=\Delta u-\nabla \cdot(u(\nabla K * u)),
$$

for various kernels $K$ is considered. The existence results are given for two kinds of kernels: mildly singular (such that $\nabla K \in L^{q^{\prime}}\left(\mathbb{R}^{n}\right)$ for some $q^{\prime} \in(n, \infty]$ ) and strongly singular (if $\nabla K \in L^{q^{\prime}}\left(\mathbb{R}^{n}\right)$ for some $q^{\prime} \in(1, n]$ and $\nabla K \notin L^{p}\left(\mathbb{R}^{n}\right)$ for every $p>n$ ). Since the kernel $K$ (given by (1.2)) is an example of a strongly singular kernel, under smallness assumption on $\left\|u_{0}\right\|_{1}$, Theorem 2.6 in [18] implying global existence and decay as in [21] can be applied.

As far as we know, there is no result (except that in [21] and [18] contained in the existence results) concerning the asymptotic behavior of the solution to the considered problem. The problem of the asymptotic profile for the problem with parabolic version of (1.2) was studied in the papers [20,25] and [26], under the general assumption that $L^{1}$ and $L^{\infty}$ norms of solutions are bounded. Assuming additionally boundedness of the quantities $\int u \nabla v$ and $\int y u_{0}(y) d y$, the asymptotics of the solutions is given by the heat kernel $G(t)$. For $n \geq 2$, the solution to the problem behaves in the $L^{p}$ norm for $t \rightarrow+\infty$ as $G(t) \int u_{0}(y) d y+\left(\int y u_{0}(y) d y+\int_{0}^{t} \int u \nabla v d y d \tau\right) \cdot \nabla G(t)$ with the rate $t^{-\frac{n}{2}\left(1-\frac{1}{p}\right)-\frac{1}{2}}$. For $n=1$, an analogous result required an additional logarithmic decay, i.e., the quantity $t^{\frac{n}{2}\left(1-\frac{1}{p}\right)+\frac{1}{2}}$ should be replaced by $t^{\frac{1}{2}\left(1-\frac{1}{p}\right)+\frac{1}{2}}(\log t)^{-1}$.

Our main result - asymptotics of the solutions to the problem (1.1)-(1.2) - is analogous to that one obtained for the problem with the parabolic version of (1.2).

In fact, since the term $\int u \nabla v$ for the solutions of (1.1)-(1.2) is equal to 0 , our asymptotics coincide with that of the parabolic-parabolic version of the problem, i.e., the solution tends to $G(t) \int u_{0}(y) d y+\int y u_{0}(y) d y \cdot \nabla G(t)$ in $L^{p}$ norms with the rate $t^{-\left(1-\frac{1}{p}\right)-\frac{1}{2}}$.

The obtained result suggests that the heat kernel is a good approximation of the solution (at least for small masses). Such asymptotics might be expected. Applying scaling techniques [19] and introducing for $\lambda>0$ new functions $u_{\lambda}(x, t)=\lambda^{2} u\left(\lambda x, \lambda^{2} t\right)$, 
$v_{\lambda}(x, t)=v\left(\lambda x, \lambda^{2} t\right)$, the system (1.1)-(1.2) can be transformed to

$$
\begin{aligned}
\left(u_{\lambda}\right)_{t} & =\Delta u_{\lambda}-\nabla \cdot\left(u_{\lambda} \nabla v_{\lambda}\right), \\
\Delta v_{\lambda} & =\lambda^{2} v_{\lambda}-u_{\lambda} .
\end{aligned}
$$

Letting $\lambda \rightarrow+\infty$, we may expect that $v_{\lambda} \rightarrow 0$, and consistently the drift term in the parabolic equation can be negligible to get a Gaussian profile for $u$.

Analogously, a similar corollary can deduced after the transformation [6] $u=$ $\frac{1}{R(t)^{2}} \tilde{u}\left(\log R(t), \frac{x}{R(t)}\right), v=\tilde{v}\left(\log R(t), \frac{x}{R(t)}\right)$ with $R(t)=\sqrt{1+2 t}, \tau(t)=$ $\log R(t)$ applied for (1.1)-(1.2). The obtained system reads

$$
\begin{aligned}
\tilde{u}_{t} & =\Delta \tilde{u}+\nabla \cdot(\tilde{u} y)-\nabla \cdot(\tilde{u} \nabla \tilde{v}), \\
\Delta \tilde{v} & =-\tilde{u}+\alpha(\tau) \tilde{v}
\end{aligned}
$$

so $\alpha(\tau) \rightarrow+\infty$ as $t \rightarrow+\infty$. Vanishing of $\tilde{v} \rightarrow 0$ should also imply that asymptotics of $u$ is given by the heat kernel.

\section{Main result}

In this paper, we focus on mild solutions. Recall that a mild solution of problem (1.1)-(1.3) is defined usually as a solution of the following integral equation (also called the Duhamel formula)

$$
u=e^{t \Delta} u_{0}+\int_{0}^{t} \nabla e^{(t-s) \Delta}(u(\nabla K * u)) d s,
$$

where $\nabla K$ is the gradient of the two-dimensional fundamental solution of the Eq. (1.2), given by the Bessel potential.

Let us introduce functional Banach spaces relevant to the study of solutions of the Cauchy problem for the considered system, $p \in(1, \infty)$

$$
\mathcal{X}_{p}=\mathcal{C}\left([0,+\infty), L^{1}\left(\mathbb{R}^{2}\right)\right) \cap\left\{\mathcal{C}\left((0,+\infty), L^{p}\left(\mathbb{R}^{2}\right)\right) ; \sup _{t>0} t^{1-\frac{1}{p}}\|u(t)\|_{L^{p}}<+\infty\right\}
$$

The norm in the space $\mathcal{X}_{p}$ is given by

$$
\|u\|_{\mathcal{X}_{p}} \equiv\|u\|_{1}+\|u\|_{p},
$$

where

$$
\|\| u\left\|\left.\right|_{p}=\sup _{t>0} t^{1-\frac{1}{p}}\right\| u(t) \|_{L^{p}} .
$$

To simplify the notation, let us introduce also the quantity

$$
\|\mid u\|_{p}^{*}=\sup _{t>0} t^{\frac{3}{2}-\frac{1}{p}}\|u(t)\|_{L^{p}}
$$


The main aim of the paper is to establish the asymptotic profile for the system (1.1)-(1.3). In this section, we give a precise formulation of the main result, discuss its sharpness together with a comparison with analogous models, and outline the key steps of the proof of the main statement.

First, following [21], let us present the statement of the existence result

THEOREM 2.1. Let $u_{0} \in L^{1}\left(\mathbb{R}^{2}\right)$. There exists $\varepsilon>0$, such that for each $p \in\left(\frac{4}{3}, 2\right)$ and $\left\|u_{0}\right\|_{1}$ sufficiently small, there exists a unique solution to the problem (2.1) in the open ball of radius $\varepsilon$ in the space $\mathcal{X}_{p}$.

The main result describes the asymptotics of solutions for $t \rightarrow+\infty$.

THEOREM 2.2. Let $u_{0} \in L^{1}\left(\mathbb{R}^{2}\right)$ and $1 \leq p \leq+\infty$. Assume that $\int y u_{0}(y) d y$ is finite. Then, for the solution $u(t)$-obtained in Theorem 2.1, we have

$$
\lim _{t \rightarrow+\infty} t^{\frac{3}{2}-\frac{1}{p}}\left\|u(t)-M G(t)-\left(\int y u_{0}(y) d y\right) \nabla G(t)\right\|_{p}=0 .
$$

As it can be easily calculated, the center of mass $\left(\int y u(t, y) d y\right)$ is preserved. This, together with the shift invariance of the problem, allows to rewrite, without lose of generality, the main statement as

$$
\lim _{t \rightarrow \infty} t^{\frac{3}{2}-\frac{1}{p}}\|u(t)-M G(t)\|_{p}=0
$$

Since such a simplification will not give any advantage in the calculations, we leave the statement of the main result in the previous version, which also is analogous to result for the parabolic-parabolic problem.

We want to emphasize the differences in the asymptotics comparing with the model used to the description of the temporal evolution of gravitationally self-interacting particles, i.e.,

$$
\begin{aligned}
u_{t} & =\Delta u-\nabla \cdot(u \nabla v), \\
-\Delta v & =u .
\end{aligned}
$$

The intermediate asymptotics of the solution for this model is given by its self-similar solutions (see remarks below). Introduction of the degradation term in the Eq. (2.4) (namely, replacing (2.4) by $\Delta v+v=u$ ) destroys this self-similar asymptotics and implies asymptotics given by the heat kernel.

Let us compare the above result with the asymptotics of the solution to the doubly parabolic model (2.6)-(2.7) with $\varepsilon=1$. Here, we would like to emphasize that, although the decay rates of solutions in both cases are the same, in the parabolicparabolic case to obtain such a decay one needs to extract the quantity of $\nabla G(t)$ with the coefficient which strongly depends on the solution (via the term $\int_{0}^{t} \int u \nabla v$ ). In the considered parabolic-elliptic case, $\nabla G(t)$ is included in the asymptotic profile but without such a condition, the coefficient depends on $u_{0}$ only. We note additionally that 
the obtained result was proved without any assumption on $L^{\infty}$ norm of the solution, contrary to the doubly parabolic case.

For (1.1)-(1.3), i.e., assuming so-called quasi-stationary hypothesis for the chemoattractant, both equations are not so strongly coupled as in the parabolic-parabolic case. First, we solve the elliptic equation for $v$-given by the convolution with the Bessel potential $v=(-\Delta+I)^{-1} u-$ getting a nonlocal, but linear, function of $u$-and then eliminate $v$ from the first parabolic equation leaving a nonlinear nonlocal equation for $u$. This instantaneous diffusion of $v$ erases any inertial effect which would appear in the doubly parabolic case.

In the parabolic-parabolic case (considered in [20,25,26]), we have parabolic equation describing temporal evolution of $v$ and thus we need to estimate the solutions $u$ and $v$ simultaneously (getting also analogous results for decay of the function $v$ ).

Let us come back to a similar one (at first glance) model (2.3)-(2.4). The literature for this problem is much richer than for (1.1)-(1.2), and obtained results are sharper (see $[1,3,8]$ and references therein). In particular, $8 \pi$ as a critical mass was subject of intensive studies recently. Indeed, it has been proved that there exists a threshold for the initial mass. For masses under this threshold, the solution exists globally in time, while for larger than this critical mass (with an additional assumption on $u_{0}$ ), the solution blowup in a finite time (see [7]). Probably, the most complete picture of the situations for (2.3)-(2.4) is given in the papers [12] and [8] where the existence and asymptotics behavior in the energy space was considered. Applying entropy dissipations methods, the existence of global solutions (in the subcritical case, up to the mass $8 \pi$ ), as well as the convergence to self-similar solutions was proved. For related studies, we refer the reader to the above-mentioned papers and the references therein.

Recall that in the problem considered in this paper, analogously to (2.3)-(2.4), a similar threshold for mass appears. For mass greater than $8 \pi$, solutions blowup (under an additional assumption on the second moment [10, Theorem 1.2]), while for mass below this threshold, global solutions exist.

By (2.4), $v$ is given by convolution with Riesz potential. Note that Riesz and Bessel potentials are related in the following way ([28, Chap. 5, Sect. 3.2]): the operators

$$
(-\Delta)^{\frac{\alpha}{2}}(-\Delta+I)^{-\frac{\alpha}{2}}
$$

are bounded on every $L^{p}\left(\mathbb{R}^{n}\right), 1 \leq p \leq \infty, \alpha>0$. Thus, some methods used for the system (2.3)-(2.4) can be applied for (1.1)-(1.3). Indeed, such an idea was applied, for example in [21, Lemma 2.1], to get existence result.

After discussing similarities described above, let us shortly outline the differences between these models, which result in different asymptotics.

The problem (2.3)-(2.4), contrary to (1.1)-(1.2), admits the so-called self-similar solutions, i.e., functions which fulfill the following identity

$$
u(x, t)=\lambda^{2} u\left(\lambda x, \lambda^{2} t\right), \quad v(x, t)=v\left(\lambda x, \lambda^{2} t\right)
$$

for all $\lambda>0$. In the papers [7] and [8], the convergence of the solutions to selfsimilar one was proved. Note that self-similar solutions are of the form $u(x, t)=$ 
$t^{-1} u\left(\frac{x}{\sqrt{t}}, 1\right)=t^{-1} U\left(\frac{x}{\sqrt{t}}\right)$. The obvious scaling property $\|f(\lambda \cdot)\|_{p}=\lambda^{\frac{2}{p}}\|f\|_{p}$, suggests the decay of $L^{p}$ norm of solutions as $t^{-1+\frac{1}{p}}$ (compare existence results in previously discussed papers and Theorem 4.1 of this paper). Note that the system (2.3)-(2.4) is an example of a parabolic equation with quadratic nonlinearity enjoying some scaling property. In [17, Theorem 6.1], it was proved that the solutions tends to a self-similar solution (a direct application result of [17] with precise proofs can be found in [15, Corollary 5.2] for problem (2.3)-(2.4) with changed sign of (2.4)). This convergence was obtained under small masses assumption only, contrary to results in [7] (radially symmetric solutions only) and [8] (additional assumption on the quantity $\int u_{0} \log u_{0}$ and the second moment).

As we will see later, the proofs of the $t^{-1+\frac{1}{p}}$ decay of the solution are based on these properties of the kernel, shared by Bessel and Riesz potentials (the gradient of the kernel belongs to $L^{2, \infty}\left(\mathbb{R}^{2}\right)$ ). Proving the main result-Theorem 2.2-we use a property of the Bessel potential (the finite $L^{1}$ norm) which the Riesz potential does not possess.

It seems that in this aspect of behavior of solutions, the considered problem shares more properties with its parabolic-parabolic version (compare the term $\iint u \nabla v d x d t$ appearing in the asymptotics, equal to 0 for the parabolic-elliptic problem) than with the model with the Riesz potential.

Having in mind the results obtained for the problem (2.3)-(2.4), the natural question of sharpness of the result for (1.1)-(1.2) arises. The result obtained in this paper is not sharp in the sense that it gives asymptotics for solutions with small masses only, much smaller than $8 \pi$, which is known as the critical value of mass for the existence of global solutions. It seems that to obtain a sharp result, we need to develop theory based on entropy methods (which seems to be more efficient especially for masses close to the critical one) analogous to these developed for (2.3)-(2.4) ([8] and references therein). However, such results obtained in the energy space need additional assumptions on the second moment and $\int u_{0} \log u_{0}$, while in this paper, we concentrate on intermediate asymptotics of solutions under conditions on initial mass only.

Note that in [8], the intermediate asymptotics of solutions has been obtained without the decay rate, contrary to the considered case.

On the other hand, reading carefully the proof of Proposition 4.5, we can deduce that the asymptotics described by Theorem 2.2 may be extended for those initial conditions for which we can prove the $t^{-\left(1-\frac{1}{p}\right)}$ decay of $L^{p}$ norm of solutions (a similar situation appears for some models with a modification of the first parabolic equation to prevent the overcrowding of amoebae [9, Theorem 2.10]).

Finally, let us recall that the system (1.1)-(1.2) can be considered as a formal limit of the parabolic-parabolic version with time derivative of $v$ multiplied by a positive parameter $\varepsilon>0$, i.e., with the system

$$
\begin{aligned}
u_{t} & =\Delta u-\nabla \cdot(u \nabla v), \\
\varepsilon v_{t} & =\Delta v-v+u,
\end{aligned}
$$


where $\varepsilon$ expresses the relative speed of propagation of the species and the chemoattractant. For existence result, see e.g., the paper [10] where techniques used in [8] have been applied. The reader may be also interested in the paper [23] with second equation given by $\varepsilon v_{t}=\Delta v+u$ where variational methods have been applied to get self-similar solutions, or in [5] where a construction of solutions to doubly parabolic problem with mass above critical for the parabolic-elliptic $8 \pi$ was presented.

A natural assumption is that the diffusion of the chemicals takes place much faster than that of the species, so we may assume $\varepsilon \ll 1$. In view of the smallness assumption on the parameter $\varepsilon$, a natural question arises: how do behave solutions to the problem (2.6)-(2.7) when the parameter $\varepsilon$ tends to 0 , and the mentioned parabolic-parabolic problem formally converges to the parabolic-elliptic one. A natural conjecture is that solutions of doubly parabolic problem tend in a suitable sense (at least locally in $L^{1}$ ) as $\varepsilon \rightarrow 0$ to an adequate solution of the system (1.1)-(1.2) (unique and with the same initial condition $u_{0}$, small enough to guarantee global existence). Such a conjecture was proved for gravitational interactions (system (2.3)-(2.4)) in the space of pseudomeasures [27], or in a class of functions with natural space-time decay properties, i.e., such that $\sup _{t>0, x \in \mathbb{R}^{2}}\left(t+|x|^{2}\right)|u(x, t)|$ is finite [4].

Now let us present the steps in the proof of the main result. We begin with the proof of the existence of the solutions to the problem for a restricted range of $p$, i.e., we prove Theorem 2.1. Having the existence of solutions in the spaces $\mathcal{X}_{p}$, which implies the decay of the solutions for $L^{p}$ norm, $p \in\left(\frac{4}{3}, 2\right)$, we extend this range for all $p \in[1,+\infty$ ) (Proposition 4.1). Since the main result is based on estimates for the gradient of the solution, we prove an analogous result also for the gradient of the solution. We show (Proposition 4.3) that for each $p \in[1,+\infty]$ we have

$$
t^{\frac{3}{2}-\frac{1}{p}}\|\nabla u(t)\|_{p} \leq \text { const. }<\infty .
$$

Equipped with these estimates for the solution and its gradient, we will prove Theorem 2.2.

\section{Preliminaria}

Before we give the proofs of the statements from the previous section, let us recall a well-known Banach-type theorem useful in proving the existence result.

THEOREM 3.1. Let $\mathcal{X}$ be a Banach space. Assume that $B: \mathcal{X} \times \mathcal{X} \rightarrow \mathcal{X}$ is a continuous bilinear operator, i.e., there exists a constant $K$ such that

$$
\|B(y, z)\| \mathcal{X} \leq K\|y\| \mathcal{X}\|z\| \mathcal{X}
$$

for all $y, z \in \mathcal{X}$. For $a \in \mathcal{X}$ such that $\|a\|_{\mathcal{X}} \leq \frac{1}{4 K}$, there exists a solution $x \in \mathcal{X}$ to the equation

$$
x=a+B(x, x) .
$$


This solution fulfills the estimate $\|x\| \mathcal{X} \leq \frac{1-4 K\|a\| \mathcal{X}}{2 K} \leq \frac{1}{2 K}$. Additionally, this solution is unique in the open ball in $\mathcal{X}$ of radius $\frac{1}{2 K}$ centered at zero.

The proof can be found, for example, in [22] or [2, Lemma 2].

Now, let us recall a more detailed information about the kernel $K$ defining the function $v$ in (1.2). The Bessel potential is given by the integral

$$
K(x)=\gamma_{n} e^{-|x|} \int_{0}^{\infty} e^{-|x| s}\left(s+\frac{s^{2}}{2}\right)^{\frac{n-3}{2}} d s
$$

where $\gamma_{n}=(2 \pi)^{\frac{1-n}{2}}\left(2 \Gamma\left(\frac{n-1}{2}\right)\right)$. Since

$$
\nabla K(x)=\gamma_{n} \frac{x}{|x|} e^{-|x|} \int_{0}^{\infty} e^{-|x| s}\left(s+\frac{s^{2}}{2}\right)^{\frac{n-3}{2}}(1+s) d s
$$

then

$$
\begin{aligned}
& |\nabla K(x)| \sim|x|^{-1} \text { for } x \text { close to } 0, \\
& |\nabla K(x)| \sim e^{-|x|} \text { for } x \rightarrow+\infty .
\end{aligned}
$$

Observe that $\nabla K \in L^{2, \infty}$, where $L^{2, \infty}$ is the weak $L^{p}$ space (the Marcinkiewicz space), which is frequently used in the proofs. Note that, contrary to the case of the Riesz potential, $\nabla K \in L^{p}\left(\mathbb{R}^{2}\right)$ for $p<2$.

For more information about the Bessel potential, see, e.g., [28, Chap. 5, Sect. 3.1].

Finally, let us recall the following well-known estimates for the heat kernel which are consequences of the Young inequality for convolutions

$$
\begin{aligned}
& \left\|e^{t \Delta} f\right\|_{p} \leq C(p, q) t^{-\left(\frac{1}{p}-\frac{1}{q}\right)}\|f\|_{q}, \\
& \left\|\nabla e^{t \Delta} f\right\|_{p} \leq C(p, q) t^{-\left(\frac{1}{p}-\frac{1}{q}\right)-\frac{1}{2}}\|f\|_{q}
\end{aligned}
$$

valid for each $q, p$ such that $1 \leq q \leq p \leq+\infty$ and each function $f \in L^{q}\left(\mathbb{R}^{n}\right)$. The constant $C$ depends only on $p$ and $q$ and is equal to 1 in the case $q=p$.

Below, the letter $C$ denotes various inessential constants which may vary from line to line. Since we are not concentrated on the optimality of the constants, we indicate the dependence of $C$ on essential parameters only. For example, for parameters $\alpha, \beta, \ldots$, we will write $C(\alpha, \beta, \ldots)$. The integral $\int$ without any subscript denotes the integral over the whole $\mathbb{R}^{2}$.

\section{Proofs}

In this section, we give the proofs of the statements announced in the Sect. 2. 


\subsection{Existence: the proof of Theorem 2.1}

By (3.2), $e^{t \Delta} u_{0}$, the linear term in (3.1), can be estimated by

$$
\left\|e^{t \Delta} u_{0}\right\|_{p} \leq C t^{1-\frac{1}{p}}\left\|u_{0}\right\|_{1}
$$

thus we begin with the estimates for the $L^{p}$ norm of the bilinear part.

Using (3.3), we have

$$
\begin{aligned}
\|B(u, u)(t)\|_{p} & \leq C \int_{0}^{t}(t-s)^{-\left(\frac{1}{r}-\frac{1}{p}\right)-\frac{1}{2}}\|u(s)(\nabla K * u(s))\|_{r} d s \\
& \leq C \int_{0}^{t}(t-s)^{-\left(\frac{1}{r}-\frac{1}{p}\right)-\frac{1}{2}}\|u(s) * \nabla K\|_{m}\|u(s)\|_{p} d s \\
& \leq C \int_{0}^{t}(t-s)^{-\left(\frac{1}{r}-\frac{1}{p}\right)-\frac{1}{2}}\|u(s)\|_{p}\|\nabla K\|_{2, \infty}\|u(s)\|_{p} d s
\end{aligned}
$$

where

$$
\frac{1}{m}=\frac{1}{p}-\frac{1}{2}, \quad \frac{1}{r}=\frac{1}{m}+\frac{1}{p}=\frac{2}{p}-\frac{1}{2} .
$$

To guarantee the fulfillment of the condition $r, m \in(1,+\infty)$, we need to restrict the range of $p$ 's, i.e., $\frac{4}{3}<p<2$.

Using the fact that $u$ belongs to $\mathcal{X}_{p}$ space, we obtain

$$
\|B(u, u)\|\left\|_{p} \leq C \int_{0}^{t}(t-s)^{-\frac{1}{p}} s^{-2\left(1-\frac{1}{p}\right)} d s\right\| \nabla K\left\|_{2, \infty}\right\| u \|_{p}{ }^{2},
$$

which gives

$$
\left.\|B(u, u)\|\right|_{p} \leq C\|\nabla K\|_{2, \infty}\|\| u \|_{p}^{2} .
$$

Dealing with the $L^{1}$ norm, notice that repeating the above calculations for $p=1$ may lead to restrictions of the range of admissible values of $p$ up to the empty set. To preserve the same range which is possible for the $L^{p}$ norm, we have to modify the proof in the following way

$$
\begin{aligned}
\|B(u, u)(t)\|_{1} & \leq \int_{0}^{t}(t-s)^{-\frac{1}{2}}\|u(s)(\nabla K * u(s))\|_{1} d s \\
& \leq \int_{0}^{t}(t-s)^{-\frac{1}{2}}\|u(s) * \nabla K\|_{m}\|u(s)\|_{z} d s \\
& \leq C \int_{0}^{t}(t-s)^{-\frac{1}{2}}\|u(s)\|_{z}\|\nabla K\|_{2, \infty}\|u(s)\|_{p} d s,
\end{aligned}
$$

where $m, z$ are defined by

$$
\frac{1}{m}=\frac{1}{p}-\frac{1}{2}, \quad \frac{1}{z}+\frac{1}{m}=1,
$$


and imply the following restriction on $p$

$$
\frac{4}{3}<p<2
$$

Interpolating the $L^{z}$ norm of the solution by $\|u\|_{z} \leq\|u\|_{1}^{1-\theta}\|u\|_{p}^{\theta}$ with

$$
\frac{1}{z}=1-\theta+\frac{\theta}{p} \quad \text { which gives } \quad \theta=\frac{\frac{1}{p}-\frac{1}{2}}{1-\frac{1}{p}} \quad \text { and } 1+\theta=\frac{\frac{1}{2}}{1-\frac{1}{p}},
$$

we get

$$
\begin{aligned}
\|B(u, u)(t)\|_{1} & \leq C \int_{0}^{t}(t-s)^{-\frac{1}{2}}\|u(s)\|_{1}^{1-\theta}\|\nabla K\|_{2, \infty}\|u(s)\|_{p}^{1+\theta} d s \\
& \leq C \int_{0}^{t}(t-s)^{-\frac{1}{2}} s^{-\left(1-\frac{1}{p}\right)(1+\theta)} d s\|\nabla K\|_{2, \infty}\|u\|\left\|_{p}{ }^{1+\theta}\right\| u \|_{1}^{1-\theta}
\end{aligned}
$$

leading to

$$
\|B(u, u)\|_{1} \leq t^{-\frac{1}{2}-\frac{1}{2}+1} C\|\nabla K\|_{2, \infty}\|u\|_{p}{ }^{1+\theta}\|u\|_{1}^{1-\theta} \leq C\|\nabla K\|_{2, \infty}\|u\|\left\|_{p}{ }^{1+\theta}\right\| u \|_{1}^{1-\theta} .
$$

Since $\|u\| \|_{p}$ is bounded, the above estimate implies the boundedness of $\|B(u, u)\|_{1}$.

Note that for $a_{p}=\|u(t)\|_{p}$ due to the above calculations, we arrive at

$$
a_{p} \leq C_{p}+K_{p} a_{p}^{2} \text { and } a_{1} \leq C_{1}+K_{1} a_{1}^{1-\theta} a_{p}^{1+\theta} \text { for } p \in\left(\frac{4}{3}, 2\right)
$$

where $C_{p}$ and $K_{p}$ can be obtained from above. Using this dependence, we modify Theorem 3.1 in a way presented in [21] to obtain existence and uniqueness of the solutions, under smallness condition on $\left\|u_{0}\right\|_{1}$.

Granted the result on the time decay included in the definition of $\mathcal{X}_{p}$, we establish required time decay of the solutions for all indices $p$.

\subsection{The decay of the solution}

In this section, we extend the decay of the solutions obtained in the previous section to all indices $p \in[1,+\infty)$, i.e., we prove

PROPOSITION 4.1. Let $u(t)$ be the solution obtained in Theorem 2.1. For each $p \in[1,+\infty)$, we have

$$
\sup _{t>0} t^{1-\frac{1}{p}}\|u(t)\|_{p} \leq \mathrm{const}<\infty .
$$

To prove this proposition, first, we establish a sequence of indices, tending to $+\infty$, for which the required decay is true. To avoid any confusion, we denote temporarily indices greater than 2 by $q$. 
LEMMA 4.2. Let $u(t) \in \mathcal{X}_{p}$ for $p \in\left(\frac{4}{3}, 2\right)$. For $\alpha=2^{n}$, such that $\frac{\alpha}{\alpha-1}>$ $4\left(\frac{1}{p}-\frac{1}{4}\right)$, and all $n \in \mathbb{N}$, there exists a subset of $q \in\left(2^{n}, 2^{n+1}\right)$ for which

$$
\|u(t)\|_{q} \leq C t^{-\left(1-\frac{1}{q}\right)} .
$$

Proof. We begin with the estimate of the bilinear term. For $q \geq \alpha$, we have

$$
\begin{aligned}
\|B(u, u)(t)\|_{q} & \leq C \int_{0}^{t}(t-s)^{-\left(\frac{\alpha}{q}-\frac{1}{q}\right)-\frac{1}{2}}\|u(s)(\nabla K * u(s))\|_{\frac{q}{\alpha}} d s \\
& \leq C \int_{0}^{t}(t-s)^{-\frac{\alpha-1}{q}-\frac{1}{2}}\|u(s) * \nabla K\|_{m}\|u(s)\|_{p} d s \\
& \leq C \int_{0}^{t}(t-s)^{-\frac{\alpha-1}{q}-\frac{1}{2}}\|u(s)\|_{p}\|\nabla K\|_{2, \infty}\|u(s)\|_{p} d s \\
& \leq C \int_{0}^{t}(t-s)^{-\frac{\alpha-1}{q}-\frac{1}{2}} s^{-2\left(1-\frac{1}{p}\right)} d s\|\nabla K\|_{2, \infty}\|u\|_{p}{ }^{2}
\end{aligned}
$$

with

$$
\frac{1}{m}=\frac{1}{p}-\frac{1}{2} \text { and } \frac{\alpha}{q}=\frac{1}{m}+\frac{1}{p}=\frac{2}{p}-\frac{1}{2} .
$$

Restrictions imposed on $m(1<m<+\infty)$ and exponents of $s$ and $(t-s)$ are fulfilled by $p \in\left(\frac{4}{3}, 2\right)$ and $q>2(\alpha-1)$. For such $p$ and $q$, we have

$$
\|B(u, u)\|_{q} \leq t^{-\left(1-\frac{1}{q}\right)} t^{-\frac{1}{2}} t^{-\left(\frac{\alpha}{q}-\frac{2}{p}\right)} C\|\nabla K\|_{2, \infty}\|u\| \|_{p}^{2} .
$$

Note that since $p<2 \leq \alpha<q$, we cannot obtain a better decay than $t^{-\left(\frac{3}{2}-\frac{1}{q}\right)}$.

To prove the statement of the Lemma, let us choose $q$ as follows

$$
q=\alpha\left(\frac{2}{p}-\frac{1}{2}\right)^{-1}
$$

Note that for such a choice of $q$, the necessary condition $q>2(\alpha-1)$ is equivalent to

$$
\frac{\alpha}{\alpha-1}>2\left(\frac{2}{p}-\frac{1}{2}\right) .
$$

Now observe that for $p \in\left(\frac{4}{3}, 2\right)$, the range of $2\left(\frac{2}{p}-\frac{1}{2}\right)$ is $(1,2)$ then for all $\alpha$ (equivalently for all $n$ ) we can choose $p$ (also $q$, by (4.1)) such that (4.2) is satisfied. In this way, we can always find a subset of $q$ of $\left(2^{n}, 2^{n+1}\right)$ for which

$$
\left\|u(t)-e^{t \Delta} u_{0}\right\|_{q} \leq t^{-\left(1-\frac{1}{q}\right)} C\|\nabla K\|_{2, \infty}\|u\|_{p}{ }^{2},
$$

so due to the decay of the heat semigroup for $q$, we get

$$
\sup _{t>0} t^{-\left(1-\frac{1}{q}\right)}\|u(t)\|_{q} \leq C\|\nabla K\|_{2, \infty}\|\| u \|_{p}{ }^{2} .
$$


Proof. (of the Proposition 4.1). To prove Proposition 4.1 we recall that, by the definition of $X_{p}$ space and Theorem 2.1, the statement of the proposition is obviously fulfilled for $p \in\{1\} \cup\left(\frac{4}{3}, 2\right)$. Due to decay properties of the heat semigroup, we are left with the estimates for the bilinear form. By the existence result, the assumption of Lemma 4.2 is fulfilled. Applying this Lemma, we get the existence of a sequence of $q_{n}$ for which we have the required decay of $u(t)$. Since $\|u(t)\|_{1} \leq$ const and $q_{n} \rightarrow+\infty$ due to interpolation of $L^{q}$ norm, i.e., for $q<q_{n}$, $\|u(t)\|_{q} \leq C\|u(t)\|_{1}^{1-\theta}\|u(t)\|_{q_{n}}^{\theta}$ for

$$
\theta=\frac{1-\frac{1}{q}}{1-\frac{1}{q_{n}}},
$$

we get the statement of the Proposition 4.1 for all $p \in[1,+\infty)$.

4.3. The decay of the gradient of the solution

Now, we deal with the existence and decay of the gradient of $u(t)$. We prove the following:

PROPOSITION 4.3. Let $u_{0} \in L^{1}\left(\mathbb{R}^{2}\right)$ and for all $p \in(1,+\infty)$ the solution $u(t)$ is in $\mathcal{X}_{p}$. Then, for each $p \in[1,+\infty]$, the gradient of the solution $u(t)$ satisfies the following time decay

$$
t^{\frac{3}{2}-\frac{1}{p}}\|\nabla u(t)\|_{p} \leq \text { const. }<\infty
$$

Proof. Arguments similar to these in the existence section allow to show that $\nabla u \in$ $L^{p}\left(\mathbb{R}^{2}\right)$ for certain $p$. Since these calculations resemble those in the previous section, we skip the details and concentrate on boundedness of $\nabla u$. Since the main difference comparing to the method applied in the existence theorem is the form of obtained inequalities for $L^{1}$ and $L^{p}$ norms of $\nabla u$, we concentrate on that issue.

Similarly, as in the previous section, we consider separately $p$ in two cases: $p<2$ and $p>2$.

Let us begin with $p<2$ and concentrate on $\|\nabla u\|_{p}$. We split the expression for $\nabla u(t)$ as follows

$$
\nabla u(t)=\nabla e^{t \Delta} u_{0}+B_{1}+B_{2}+B_{3},
$$

where $B_{i}$ are given by

$$
\begin{aligned}
& B_{1}=\int_{0}^{\alpha t} \nabla \cdot \nabla e^{(t-s) \Delta}(u(s)(\nabla K * u(s))) d s, \\
& B_{2}=\int_{\alpha t}^{t} \nabla e^{(t-s) \Delta}(\nabla u(s)(\nabla K * u(s))) d s, \\
& B_{3}=\int_{\alpha t}^{t} \nabla e^{(t-s) \Delta}(u(s)(\nabla K * \nabla u(s))) d s
\end{aligned}
$$

for some $\alpha \in(0,1)$. 
We estimate $B_{1}$ as

$$
\begin{aligned}
\left\|B_{1}\right\|_{p} & \leq C \int_{0}^{\alpha t}(t-s)^{-2+\frac{1}{p}}\|u(s)(\nabla K * u(s))\|_{1} d s \\
& \leq C \int_{0}^{\alpha t}(t-s)^{-2+\frac{1}{p}}\|u(s) * \nabla K\|_{\varepsilon^{-1}}\|u(s)\|_{1+\eta_{1}} d s \\
& \leq C \int_{0}^{\alpha t}(t-s)^{-2+\frac{1}{p}}\|u(s)\|_{1+\eta_{1}}\|\nabla K\|_{2, \infty}\|u(s)\|_{2-\eta_{2}} d s
\end{aligned}
$$

where $\eta_{1}$ and $\eta_{2}$ are chosen such that for a given $\varepsilon$

$$
\frac{1}{1+\eta_{1}}=1-\varepsilon, \quad \frac{1}{2-\eta_{2}}=\frac{1}{2}+\varepsilon .
$$

Note that introduction of the $L^{\varepsilon^{-1}}$ norm is necessary, since the weak Young inequality for convolutions is not valid for, natural in this case, $L^{\infty}$ norm. Since we are separated from $t$, we are not restricted by the value of the exponent of $(t-s)$. Thus, using the fact that $u$ belongs to $\mathcal{X}_{p}$ space and interpolating the norms by $\|u\|_{1}$ and $\|u\| \|_{2}$, we get for all $p \geq 1$

$$
\begin{aligned}
\left\|B_{1}\right\|_{p} & \leq C \int_{0}^{\alpha t}(t-s)^{-2+\frac{1}{p}} s^{-\frac{1}{2}} d s\|\nabla K\|_{2, \infty}\|u\|_{1}\|u\|_{2} \\
& \leq C t^{-\left(1-\frac{1}{p}\right)-\frac{1}{2}} \int_{0}^{\alpha}(1-z)^{-2+\frac{1}{p}} z^{-\frac{1}{2}} d z\|\nabla K\|_{2, \infty}\|u\|_{1}\|u\|_{2} \\
& \leq C t^{-\left(\frac{3}{2}-\frac{1}{p}\right)}\|\nabla K\|_{2, \infty}\|u\|_{1}\|u\|_{2} C \alpha^{\frac{1}{2}}(1-\alpha)^{-2+\frac{1}{p}}
\end{aligned}
$$

So we obtain

$$
t^{\frac{3}{2}-\frac{1}{p}}\left\|B_{1}\right\|_{p} \leq C_{1}\left(\alpha,\left\|u_{0}\right\|_{1}\right)
$$

where $C_{1}$ is bounded for any $\alpha \in(0,1)$.

Next, we consider $B_{2}$. Choosing $\eta_{1}, \eta_{2}$ such that for given $\varepsilon$ and $p$

$$
\frac{1}{p-\eta_{1}}=\frac{1}{p}+\varepsilon, \quad \frac{1}{2-\eta_{2}}=\frac{1}{2}+\varepsilon,
$$

we have

$$
\begin{aligned}
\left\|B_{2}\right\|_{p} & \leq C \int_{\alpha t}^{t}(t-s)^{-\frac{1}{2}-\varepsilon}\|\nabla u(s)(\nabla K * u(s))\|_{p-\eta_{1}} d s \\
& \leq C \int_{\alpha t}^{t}(t-s)^{-\frac{1}{2}-\varepsilon}\|u(s) * \nabla K\|_{\varepsilon^{-1}}\|\nabla u(s)\|_{p} d s \\
& \leq C \int_{\alpha t}^{t}(t-s)^{-\frac{1}{2}-\varepsilon}\|\nabla u(s)\| p\|\nabla K\|_{2, \infty}\|u(s)\|_{2-\eta_{2}} d s \\
& \leq C t^{-\left(\frac{3}{2}-\frac{1}{p}\right)} \int_{\alpha}^{1}(1-z)^{-\frac{1}{2}-\varepsilon} z^{-\left(1-\frac{1}{p}\right)-\frac{1}{2}-\frac{1}{2}+\varepsilon} d s\|\nabla u\|_{p}^{*}\|\nabla K\|_{2, \infty}\|u(s)\|_{2-\eta_{2}} .
\end{aligned}
$$


Since we are separated from zero, no restriction on $p$, appearing in the exponent of $s$, should be imposed. Thus, for all $p>1$, we have

$t^{\frac{3}{2}-\frac{1}{p}}\left\|B_{2}\right\|_{p} \leq C\|\| \nabla u\left\|_{p}^{*}\right\| \nabla K\left\|_{2, \infty}\right\|\|u(s)\|_{2-\eta_{2}} 2(1-\alpha)^{\frac{1}{2}} \leq C_{2}\left(\alpha,\left\|u_{0}\right\|_{1}\right)\|\nabla u\| \|_{p}^{*}$,

where $C_{2}$ tends to 0 when $\alpha$ approaches to 1 .

Dealing with $B_{3}$ for $p \in(1,2]$, we estimate

$$
\begin{aligned}
\left\|B_{3}\right\|_{p} & \leq C \int_{\alpha t}^{t}(t-s)^{-\frac{1}{2}-\varepsilon}\|u(s)(\nabla K * \nabla u(s))\|_{p-\eta_{1}} d s \\
& \leq C \int_{\alpha t}^{t}(t-s)^{-\frac{1}{2}-\varepsilon}\|\nabla u(s) * \nabla K\|_{m}\|u(s)\|_{2-\eta_{2}} d s \\
& \leq C \int_{\alpha t}^{t}(t-s)^{-\frac{1}{2}-\varepsilon}\|\nabla u(s)\|_{p}\|\nabla K\|_{2, \infty}\|u(s)\|_{2-\eta_{2}} d s,
\end{aligned}
$$

where $\eta_{1}, \eta_{2}, m$ and $\varepsilon$ fulfill

$$
\frac{1}{p-\eta_{1}}=\frac{1}{p}+\varepsilon, \quad \frac{1}{m}=\frac{1}{p}-\frac{1}{2}, \quad \frac{1}{2-\eta_{2}}=\frac{1}{2}+\varepsilon .
$$

Note that the condition $m \leq+\infty$ implies that $p$ has to be less or equal to 2 .

Dealing exactly with the same way as with $B_{2}$ we arrive at $t^{\frac{3}{2}-\frac{1}{p}}\left\|B_{3}\right\|_{p} \leq C\|\mid \nabla u\|_{p}^{*}\|\nabla K\|_{2, \infty}\|\| u(s)\left\|_{2-\eta_{2}} 2(1-\alpha)^{\frac{1}{2}} \leq C_{3}\left(\alpha,\left\|u_{0}\right\|_{1}\right)\right\|\|\nabla u\|_{p}^{*}$, where $C_{3}$ tends to 0 when $\alpha$ goes to 1 .

Since $\left\|\nabla e^{t \Delta} u_{0}\right\|_{p} \leq C_{0}\left(\left\|u_{0}\right\|_{1}\right) t^{-\left(\frac{3}{2}-\frac{1}{p}\right)}$, we get the following inequality for $\|\mid \nabla u\|_{p}^{*}$

$$
\left\|\left|\nabla u\left\|_{p}^{*} \leq C_{0}\left(\left\|u_{0}\right\|_{1}\right)+C_{1}\left(\alpha,\left\|u_{0}\right\|_{1}\right)+\left(C_{2}\left(\alpha,\left\|u_{0}\right\|_{1}\right)+C_{3}\left(\alpha,\left\|u_{0}\right\|_{1}\right)\right)\right\|\right| \nabla u\right\|_{p}^{*},
$$

where $C_{1}$ is bounded as a function of $\alpha$, while $C_{2}$ and $C_{3}$ tend to 0 as $\alpha$ goes to 1 . Thus, for $\alpha$ such that $C_{2}+C_{3}<1$, we get a priori estimate for $\|\nabla u\|_{p}^{*}$.

Note that the estimate of $B_{1}$ is also valid for $p=1$, so we are left with the estimates of the $L^{1}$ norm of $B_{2}$ and $B_{3}$. We base the calculations for $\|u\|_{1}$ on those in the existence section. For $B_{2}$, we get

$$
\begin{aligned}
\left\|B_{2}\right\|_{1} & \leq \int_{\alpha t}^{t}(t-s)^{-\frac{1}{2}}\|\nabla u(s)(\nabla K * u(s))\|_{1} d s \\
& \leq C \int_{\alpha t}^{t}(t-s)^{-\frac{1}{2}} s-\left(1-\frac{1}{p}\right)(1+\theta)-\frac{1}{2} d s\|\nabla K\|_{2, \infty}\|\nabla u\|_{p}^{* \theta}\|\nabla u\|_{1}^{* 1-\theta}\|\| u \|_{p}
\end{aligned}
$$

leading to

$$
\left\|B_{2}\right\|_{1} \leq t^{-\frac{1}{2}} C\|\nabla K\|_{2, \infty}\left\|\left|\nabla u\left\|_{p}^{* \theta}\right\|\right| \nabla u\right\|_{1}^{* 1-\theta}\|\| u \|_{p},
$$

valid for $p \in\left(\frac{4}{3}, 2\right)$ and $\theta$ as in Sect. 4.1. 
Similar calculations give

$$
\begin{aligned}
\left\|B_{3}\right\|_{1} & \leq \int_{\alpha t}^{t}(t-s)^{-\frac{1}{2}}\|u(s)(\nabla K * \nabla u(s))\|_{1} d s \\
& \leq C \int_{\alpha t}^{t}(t-s)^{-\frac{1}{2}} s^{-\left(1-\frac{1}{p}\right)(1+\theta)-\frac{1}{2}} d s\|\nabla K\|_{2, \infty}\|u\|\left\|_{p}^{\theta}\right\| u\left\|_{1}^{1-\theta}\right\| \mid \nabla u \|_{p}^{*}
\end{aligned}
$$

leading to

$$
\left\|B_{3}\right\|_{1} \leq t^{-\frac{1}{2}} C\|\nabla K\|_{2, \infty}\|u\|_{p}^{\theta}\|u\|_{1}^{1-\theta}\|\mid \nabla u\|_{p}^{*}
$$

as $B_{2}$ valid for $p \in\left(\frac{4}{3}, 2\right)$ and $\theta$ as in Sect. 4.1.

Denoting $a_{p}=\|u(t)\|_{p}$ and $b_{p}=\|u(t)\|_{p}^{*}$, we summarize above estimates as follows

$$
b_{p} \leq C_{p}\left(a_{1}, a_{2}\right)+K_{p}\left(a_{1}, a_{2}, a_{p}\right) b_{p}
$$

with $K_{p}$ less than 1 , and

$$
b_{1} \leq C_{1}\left(b_{p}, a_{1}, a_{2}, a_{p}\right)+K_{1}\left(b_{p}^{\theta}, a_{p}\right) b_{1}^{1-\theta} \text { for } p \in\left(\frac{4}{3}, 2\right),
$$

where $C_{p}$ and $K_{p}$ can be calculated from above.

Granted the result from the Sect. 4.1 and modifying Theorem 3.1, we get the existence of the gradient and boundedness of $\|\mid \nabla u\| \|_{p}^{*}$, for small values of $\left\|u_{0}\right\|_{1}$ and $p \in[1,2)$.

To prove a similar result for $p>2$, note that we are left with estimates for $\left\|\mid B_{3}\right\| \|_{p}^{*}$ only. Since for $p<2$ we have $\|\nabla u\|_{p}^{*} \leq$ const, we repeat the calculations in Lemma 4.2 replacing $\nabla K * u$ by $\nabla K * \nabla u$ to get for $q>2$

$$
\begin{aligned}
\left\|B_{3}(u, u)(t)\right\|_{q} & \leq C \int_{\alpha t}^{t}(t-s)^{-\left(\frac{\alpha}{q}-\frac{1}{q}\right)-\frac{1}{2}}\|u(s)(\nabla K * \nabla u(s))\|_{\frac{q}{\alpha}} d s \\
& \leq C \int_{\alpha t}^{t}(t-s)^{-\frac{\alpha-1}{q}-\frac{1}{2}} s^{-2\left(1-\frac{1}{p}\right)-\frac{1}{2}} d s\|\nabla K\|_{2, \infty}\|u\|\left\|_{p}\right\| \nabla u \|_{p}^{*} \\
& \leq t^{-\left(1-\frac{1}{q}\right)-\frac{1}{2}} t^{-\left(\frac{\alpha}{q}-\frac{2}{p}\right)} C\|\nabla K\|_{2, \infty}\|u\|_{p}\|\nabla u\|_{p}^{*}
\end{aligned}
$$

for $p \in\left(\frac{4}{3}, 2\right)$ and $\alpha=2^{n}$. Note that, unlike in Lemma 4.2, the exponent of $s$ can be chosen arbitrarily.

Repeating the idea presented in the proof of Lemma 4.2, we get a sequence $q_{n} \rightarrow$ $+\infty$ for which $\left\|B_{3}\right\|_{q_{n}}$ is estimated in a required way. Interpolating the $L^{q}$ norm and letting $n \rightarrow+\infty$, we get for all $p \in(2,+\infty)$

$$
t^{\frac{3}{2}-\frac{1}{p}}\left\|B_{3}\right\|_{p} \leq C_{4}\left(\alpha,\left\|u_{0}\right\|_{1}\right)
$$

where $C_{4}$ is bounded for all $\alpha$. 
Thus for $p \in(2,+\infty)$, we obtain the following inequality

$$
\|\nabla \nabla\|_{p}^{*} \leq C_{0}\left(\left\|u_{0}\right\|_{1}\right)+C_{1}\left(\alpha,\left\|u_{0}\right\|_{1}\right)+C_{4}\left(\alpha,\left\|u_{0}\right\|_{1}\right)+C_{2}\left(\alpha,\left\|u_{0}\right\|_{1}\right)\|\nabla u\|_{p}^{*},
$$

where $C_{1}$ and $C_{4}$ are bounded for any $\alpha \in(0,1)$ and $C_{2}$ tends to 0 as $\alpha$ goes to 1 . Repeating the argument from the previous case, we get the required result.

\subsection{Proof of Theorem 2.2}

To complete the proof, recall the asymptotic behavior of the heat semigroup given by the following Lemma ([13, Theorem 4], [14])

LEMMA 4.4. Let $n \geq 1,1 \leq p \leq+\infty$ and $f \in L^{1}\left(\mathbb{R}^{n}\right)$. Then,

$$
\lim _{t \rightarrow+\infty} t^{\frac{n}{2}\left(1-\frac{1}{p}\right)}\left\|e^{t \Delta} f-\left(\int f(y) d y\right) G(t)\right\|_{p}=0 .
$$

Furthermore, under the condition $|x| f \in L^{1}\left(\mathbb{R}^{n}\right)$, it holds that

$$
\begin{aligned}
& \sup _{t>0} t^{\frac{n}{2}\left(1-\frac{1}{p}\right)+\frac{1}{2}}\left\|e^{t \Delta} f-\left(\int f(y) d y\right) G(t)\right\|_{p}<+\infty, \\
& \lim _{t \rightarrow+\infty} t^{\frac{n}{2}\left(1-\frac{1}{p}\right)+\frac{1}{2}}\left\|e^{t \Delta} f-\left(\int f(y) d y\right) G(t)+\left(\int y f(y) d y\right) \cdot \nabla G(t)\right\|_{p}=0 .
\end{aligned}
$$

Proof. The statements of the Lemma are given in Theorem 4 in [13]. The last one is fulfilled for functions $f$ with second momentum bounded. To avoid such an assumption, we obtain this relation as a limits of the compactly supported functions $\varphi_{\varepsilon}$ such that $\left\|f-\varphi_{\varepsilon}\right\|_{p}<\varepsilon,\left\|f-\varphi_{\varepsilon}\right\|_{L^{p}(|x| d x)}<\varepsilon$-which are dense in $L^{p}\left(\mathbb{R}^{2}\right)$ space-and standard approximation techniques.

Applying Lemma 4.4 for $f=u_{0}$ and $n=2$, we get

$$
\lim _{t \rightarrow \infty} t^{\frac{3}{2}-\frac{1}{p}}\left\|e^{t \Delta} u_{0}-M G(t)-\int y u_{0}(y) d y \cdot \nabla G(t)\right\|_{p}=0 .
$$

Since $u(t)=e^{t \Delta}-B(u, u)$, to prove the statement of the Theorem 2.2, we are left with time behavior of $B(u, u)$. We prove it in the following proposition:

PROPOSITION 4.5. Let $1 \leq p \leq+\infty$. Assume there exist $p_{0}$ and $q_{0}$ in $(1,+\infty)$ such that

$$
\left.\|u(t)\|\right|_{p_{0}}+\|\mid \nabla u(t)\|_{q_{0}}^{*}<\text { const. }
$$

Then,

$$
\lim _{t \rightarrow+\infty} t^{\frac{3}{2}-\frac{1}{p}}\|B(u, u)(t)\|_{p}=0 .
$$


Proof. Note that by the symmetry property of the integral defining $u(\nabla K * u)$ and due to antisymmetric property of $\nabla K$ we have $\int u(y, s)(\nabla K * u)(y, s) d y=0$, so we can write $B(u, u)$ as

$$
\begin{aligned}
B(u, u)(t) & =B(u, u)(t)-\nabla G(t) \cdot \int_{0}^{\alpha t} \int u(y, s)(\nabla K * u)(y, s) d y d s \\
& =R_{1}+R_{2}+R_{3}+R_{4}
\end{aligned}
$$

where $\alpha$ is any positive constant and $R_{i}$ is defined as follows:

$$
\begin{aligned}
& R_{1}=\int_{\alpha t}^{t} \int_{0} \nabla(x-y, t-s) \cdot u(y, s)(\nabla K * u)(y, s) d y d s, \\
& R_{2}=-\int_{0}^{\alpha t} \int_{|y| \leq \alpha \sqrt{t}}(\nabla G(x-y, t-s)-\nabla G(x, t)) \cdot u(y, s)(\nabla K * u)(y, s) d y d s, \\
& R_{3}=\int_{0}^{\alpha t} \int_{|y| \geq \alpha \sqrt{t}} \nabla G(x-y, t-s) \cdot u(y, s)(\nabla K * u)(y, s) d y d s, \\
& R_{4}=-\int_{0}^{\alpha t} \int_{|y| \geq \alpha \sqrt{t}} \nabla G(x, t) \cdot u(y, s)(\nabla K * u)(y, s) d y d s .
\end{aligned}
$$

Due to the weak Young inequality for the convolution, for $p \in[1,2)$, we estimate $R_{1}$ as

$$
\begin{aligned}
\left\|R_{1}\right\|_{p} & \leq C \int_{\alpha t}^{t}(t-s)^{-\frac{3}{2}+\frac{1}{p}}\|u(s)(\nabla K * u(s))\|_{1} d s \\
& \leq C \int_{\alpha t}^{t}(t-s)^{-\frac{3}{2}+\frac{1}{p}}\|\nabla u(s) * K\|_{p_{0}}\|u(s)\|_{q_{0}} d s \\
& \leq C \int_{\alpha t}^{t}(t-s)^{-\frac{3}{2}+\frac{1}{p}} s^{-\frac{3}{2}} d s\|\nabla u\|_{p_{0}}^{*}\|u\|_{q_{0}}\|K\|_{1},
\end{aligned}
$$

with $p_{0}, q_{0}$ fulfilling $\frac{1}{p_{0}}+\frac{1}{q_{0}}=1$. Since we are separated from zero, we have no restriction on the exponent of $s$, thus for all $p \in[1,2)$ we have

$$
t^{\frac{3}{2}-\frac{1}{p}}\left\|R_{1}\right\|_{p} \leq C t^{-\frac{1}{2}}\|\mid \nabla u\|_{p_{0}}^{*}\|u(s)\|\left\|_{q_{0}}\right\| K \|_{1} 2(1-\alpha)^{\frac{1}{p}-\frac{1}{2}} \alpha^{-\frac{3}{2}}=C_{1}(\alpha) t^{-\frac{1}{2}} .
$$

For $p>2$, we modify the above calculations in the following way

$$
\begin{aligned}
\left\|R_{1}\right\|_{p} & \leq C \int_{\alpha t}^{t}(t-s)^{-\left(\frac{1}{2}-\frac{1}{p}\right)-\frac{1}{2}+\varepsilon}\|u(s)(\nabla K * u(s))\|_{2+\eta} d s \\
& \leq C \int_{\alpha t}^{t}(t-s)^{-\left(\frac{1}{2}-\frac{1}{p}\right)-\frac{1}{2}+\varepsilon}\|\nabla u(s) * K\|_{p_{0}}\|u(s)\|_{q_{0}} d s \\
& \leq C \int_{\alpha t}^{t}(t-s)^{-1+\frac{1}{p}+\varepsilon} s^{-2-\varepsilon} d s\|\nabla u\|_{p_{0}}^{*}\|\| u\left\|_{q_{0}}\right\| K \|_{1} \\
& \leq C t^{-\left(\frac{3}{2}-\frac{1}{p}\right)} t^{-\frac{1}{2}}\|\mid \nabla u\|_{p_{0}}^{*}\|\| u \|_{q_{0}},
\end{aligned}
$$


where $\frac{1}{p_{0}}+\frac{1}{q_{0}}=\frac{1}{2+\eta}=\frac{1}{2}-\varepsilon$. The constant $\varepsilon$ we choose in such a way that $\varepsilon<\frac{1}{2}-\frac{1}{p}$, which, since there are no restrictions on the exponent of $s$, gives the required estimate.

To get the estimate of $L^{p}$ norm of $R_{2}$, first let us observe that by direct calculations, we can obtain following estimate for the integrand

$$
\|\nabla G(x-y, t-s)-\nabla G(x, t)\|_{p} \leq C\left(t^{-\left(1-\frac{1}{p}\right)-1}|y|+t^{-\left(1-\frac{1}{p}\right)-\frac{3}{2}} s\right) .
$$

Additionally, we can estimate the integral $\int_{0}^{+\infty}\|u(s)(\nabla K * u)(s)\|_{1} d s$ as

$$
\begin{aligned}
& \int_{0}^{+\infty}\|u(s)(\nabla K * u)(s)\|_{1} d s \leq \int_{0}^{1}\|u(s)(\nabla K * u)(s)\|_{1} d s \\
& \quad+\int_{1}^{+\infty}\|u(s)(\nabla K * u)(s)\|_{1} d s \leq C\left(\int_{0}^{1}\|u(s)\|_{1}^{1-\theta}\|\nabla K\|_{2, \infty}\|u(s)\|_{w}^{1+\theta} d s\right. \\
& \left.\quad+\int_{1}^{+\infty}\|u(s)\|_{q_{0}}\|\nabla u(s)\|_{p_{0}} d s\|K\|_{1}\right) \\
& \leq C\left(\int_{0}^{1} s^{-\frac{1}{2}} d s\|\nabla K\|_{2, \infty}\|u\|\left\|_{w}^{1+\theta}\right\| u\left\|_{1}^{1-\theta}+\int_{1}^{+\infty} s^{-\frac{3}{2}} d s\right\| u\left\|_{q_{0}}\right\| \nabla u\left\|_{p_{0}}^{*}\right\| K \|_{1}\right) \\
& \leq C\left(\|u\|_{w}^{1+\theta}\|u\|_{1}^{1-\theta}+\|u\|_{q_{0}}\|\nabla u\|_{p_{0}}^{*}\right),
\end{aligned}
$$

with any $w \in\left(\frac{4}{3}, 2\right)$ and $\theta$ as in Theorem 2.1 and $\frac{1}{p_{0}}+\frac{1}{q_{0}}=1$.

Applying the above estimate and the inequality (4.3), we have

$$
\begin{aligned}
\left\|R_{2}\right\|_{p} & \leq \int_{0}^{\alpha t} \int_{|y| \leq \alpha \sqrt{t}}\|\nabla G(x-y, t-s)-\nabla G(x, t)\|_{p}|u(y, s)(\nabla K * u)(y, s)| d y d s \\
& \leq t^{-\left(\frac{3}{2}-\frac{1}{p}\right)} \int_{0}^{\alpha t} \int_{|y| \leq \alpha \sqrt{t}} C\left(t^{-\frac{1}{2}}|y|+t^{-1} s\right)|u(y, s)(\nabla K * u)(y, s)| d y d s \\
& \leq t^{-\left(\frac{3}{2}-\frac{1}{p}\right)} C \alpha \int_{0}^{\alpha t}\|u(s)(\nabla K * u)(s)\|_{1} d s
\end{aligned}
$$

so that

$$
t^{\frac{3}{2}-\frac{1}{p}}\left\|R_{2}\right\|_{p} \leq \alpha C\left(\|u\|\left\|_{p}{ }^{1+\theta}\right\| u\left\|_{1}^{1-\theta}+\right\|\|u\|\left\|_{q_{0}}\right\| \nabla u \|_{p_{0}}^{*}\right)=C_{2}(\alpha) .
$$

Dealing with $R_{3}$ and $R_{4}$, we will focus on the dependence on $t$. We have

$$
\begin{aligned}
\left\|R_{3}\right\|_{p} & \leq \int_{0}^{\alpha t}\left\|\int_{|y| \geq \alpha \sqrt{t}} \nabla G(x-y, t-s) \cdot u(y, s)(\nabla K * u)(y, s) d y\right\|_{p} d s \\
& \leq C \int_{0}^{\alpha t}(t-s)^{-\left(\frac{3}{2}-\frac{1}{p}\right)} \int_{|y| \geq \alpha \sqrt{t}}|u(s)(\nabla K * u(s))| d y d s \\
& \leq C t^{-\left(\frac{3}{2}-\frac{1}{p}\right)}(1-\alpha)^{-\left(\frac{3}{2}-\frac{1}{p}\right)} \int_{0}^{\alpha t} \int_{|y| \geq \alpha \sqrt{t}}|u(s)(\nabla K * u(s))| d y d s .
\end{aligned}
$$


As we showed in the previous case, the last integral extended to the whole $\mathbb{R} \times \mathbb{R}^{2}$ is finite. Thus, this integral can be estimated by

$\int_{0}^{\alpha t} \int_{|y| \geq \alpha \sqrt{t}}|u(s)(\nabla K * u(s))| d s \leq \int_{0}^{\infty} \int_{|y| \geq \alpha \sqrt{t}}|u(s)(\nabla K * u(s))| d s=F(\alpha, t)$,

where the function $F(\alpha, t)$ for each fixed $\alpha$ tends to 0 together with $t$. So

$$
t^{\frac{3}{2}-\frac{1}{p}}\left\|R_{3}\right\|_{p} \leq C_{3}(\alpha) F(\alpha, t) .
$$

Similarly we deal with $R_{4}$ to get

$$
\left\|R_{4}\right\|_{p} \leq C t^{-\left(\frac{3}{2}-\frac{1}{p}\right)} \int_{0}^{\infty} \int_{|y| \geq \alpha \sqrt{t}}|u(y, s)(\nabla K * u)(y, s)| d y d s,
$$

and finally

$$
t^{\frac{3}{2}-\frac{1}{p}}\left\|R_{4}\right\|_{p} \leq C_{4}(\alpha) F(\alpha, t)
$$

Coming back to the statement of the proposition, we arrive at

$$
t^{\frac{3}{2}-\frac{1}{p}}\|B(u, u)(t)\|_{p} \leq C_{1}(\alpha) t^{-\frac{1}{2}}+\left(C_{3}(\alpha)+C_{4}(\alpha)\right) F(\alpha, t)+C_{2}(\alpha),
$$

where $C_{1}, C_{3}, C_{4}$ are bounded for all $\alpha, C_{2}(\alpha)$ tends to 0 together with $\alpha$, and the function $F(\alpha, t)$ tends for all $\alpha$ to 0 when $t$ tends to $+\infty$. Thus, for any $\varepsilon>0$, we can choose $\alpha_{0}$ such that $C_{2}\left(\alpha_{0}\right)<\frac{\varepsilon}{2}$. For such $\alpha_{0}$, we can choose $t_{0}$ such that for $t>t_{0}$ we have $C_{1}(\alpha) t^{-\frac{1}{2}}+\left(C_{3}(\alpha)+C_{4}(\alpha)\right) F(\alpha, t)<\frac{\varepsilon}{2}$, which implies the required statement.

\section{Acknowledgments}

The author would like to express his gratitude to G. Karch for many stimulating discussions. It is also the pleasure to thank the referee for pertinent remarks. The preparation of this paper was partially supported by the MNiSzW grant no. N N201 418839.

Open Access. This article is distributed under the terms of the Creative Commons Attribution Noncommercial License which permits any noncommercial use, distribution, and reproduction in any medium, provided the original author(s) and source are credited.

\section{REFERENCES}

[1] P. Biler, Existence and nonexistence of solutions for a model of gravitational interaction of particles, III, Colloq. Math. 68 (1995), 229-239.

[2] P. Biler, The Cauchy problem and self-similar solution for a nonlinear parabolic equation, Studia Math. 114 (1995), 181-205.

[3] P. Biler, Local and global solvability of some parabolic systems modelling chemotaxis, Adv. Math. Sci. Appl. 8 (1998), 715-743. 
[4] P. Biler, L. Brandolese, On the parabolic-elliptic limit of the doubly parabolic Keller-Segel system modelling chemotaxis, Studia Math. 193 (2009), 241-261.

[5] P. Biler, L. Corrias, J. Dolbeault, Large mass self-similar solutions of the parabolic-parabolic Keller-Segel model of chemotaxis, 1-32, J. Math. Biol. doi:10.1007/s00285-010-0357-5.

[6] P. Biler, J. Dolbeault, Long time behavior of solutions of Nernst-Planck and Debye-Hückel driftdiffusion systems, Ann. Henri Poincar, 1(3) (2000), 461-472.

[7] P. Biler, G. Karch, P. Laurençot, T. Nadzieja, The $8 \pi$-problem for radially symmetric solutions of a chemotaxis model in the plane, Math. Meth. Appl. Sci. 29 (2006), 1563-1583.

[8] A. Blanchet, J. Dolbeault, B. Perthame, Two dimensional Keller-Segel model: optimal critical mass and qualitative properties of solutions, Electron. J. Differential Equations 44, (2006), 1-32 (electronic).

[9] M. Burger, M. Di Francesco, Y. Dolak-Struss, The Keller-Segel model for chemotaxis with prevention of overcrowding: linear vs. nonlinear diffusion, SIAM J. Math. Anal. 38 (2006), 1288-1315.

[10] V. Calvez, L. Corrias, The parabolic-parabolic Keller-Segel model in $\mathbb{R}^{2}$, Comm. Math. Sci. 6 (2) (2008), 417-447.

[11] J. I. Diaz, T. Nagai, J.-M. Rakotoson, Symmetrization techniques on unbounded domains: application to a chemotaxis system on $\mathbb{R}^{N}$, J. Differential Equations 145 (1998), 156-183.

[12] J. Dolbeault, B. Perthame, Optimal critical mass in the two dimensional Keller-Segel model in $\mathbb{R}^{2}$, C. R. Acad. Sci. Paris, Ser. I 339 (2004), 611-616.

[13] J. Duoandikoetxea, E. Zuazua, Moments, masses de Dirac et decomposition de fonctions, C. R. Acad. Sci. Paris. Math. 315 (6) (1992), 693-698.

[14] M. Escobedo, E. Zuazua, Large time behavior for convection-diffusion equation in $\mathbb{R}^{n}$, J. Funct. Anal. 100 (1991), 119-161.

[15] A. Herczak, M. Olech, Existence and asymptotics of solutions of the Debye-Nernst-Planck system in $\mathbb{R}^{2}$, Banach Center Publ. 86 (2009), 129-148.

[16] W. Jäger, S. Luckhaus, On explosions of solutions to a system of partial differential equations modelling chemotaxis, Trans. Amer. Math. Soc. 329 (1992), 819-824.

[17] G. Karch, Scaling in nonlinear parabolic equations, J. Math. Anal. Appl. 234 (1999), 534-558.

[18] G. Karch, K. Suzuki, Blow-up versus global existence of solutions to aggregation equation with diffusion, (2009), 1-16, arXiv:1004.4021.

[19] G. Karch, K. Suzuki, Spikes and diffusion waves in one-dimensional model of chemotaxis, Nonlinearity 23 (2010), 1-24, arXiv:1008.0020.

[20] M. Kato, Sharp asymptotics for a parabolic system of chemotaxis in one space dimension, Diff. Integral. Eq. 22 (2009), 35-51.

[21] H. Kozono, Y. Sugiyama, Local existence and finite time blow-up of solutions in the 2-D Keller-Segel system, J. Evol. Equ. 8 (2008), 353-378.

[22] P. G. Lemarié-Rieusset, Recent Development in the Navier-Stokes Problem, Chapman \& Hall/CRC Press, Boca Raton, 2002.

[23] Y. Mizutani, N. Muramoto, K. Yoshida, Self-similar radial solutions to a parabolic system modelling chemotaxis via variational method, Hiroshima Math. J. 29 (1999), 145-160.

[24] T. Nagai, Blow-up of radially symmetric solutions to a chemotaxis system, Adv. Math. Sci. Appl. 5 (1995), 581-601.

[25] T. Nagai, R. Syukuinn, M. Umesako, Decay properties and asymptotic profiles of bounded solutions to a parabolic system of chemotaxis in $\mathbb{R}^{n}$, Funk. Ekvacioj 46 (2003), 383-407.

[26] T. Nagai, T. Yamada, Large time behavior of bounded solutions to a parabolic system of chemotaxis in the whole space, J. Math. Anal. Appl. 336 (2007), 704-726.

[27] A. Raczyński, Stability property of the two-dimensional Keller-Segel model, Asymptotic Analysis 61 (2009), 35-59.

[28] E. M. Stein, Singular Integrals and Differentiability Properties of Functions, Princeton Mathematical Series 30, Princeton University Press, Princeton, NJ, 1970.

[29] G. Wolansky, On the evolution of self-interacting clusters and applications to semilinear equations with exponential nonlinearity, J. Anal. Math. 59 (1992), 251-272. 


\author{
A. Raczyński \\ Instytut Matematyczny, \\ Uniwersytet Wroctawski, \\ pl. Grunwaldzki 2/4, 50-384 Wroctaw, \\ Poland \\ E-mail:Andrzej.Raczynski@math.uni.wroc.pl
}

